\title{
Foraging behaviour of an egg parasitoid exploiting plant volatiles induced by pentatomids: the role of adaxial and abaxial leaf surfaces
}

Francesca Frati ${ }^{\text {Corresp.. }}{ }^{1}$, Antonino Cusumano ${ }^{2}$, Eric Conti ${ }^{1}$, Stefano Colazza ${ }^{3}$, Ezio Peri ${ }^{3}$, Salvatore Guarino

${ }^{3}$, Letizia Martorana ${ }^{3}{ }^{\text {, Roberto Romani }}{ }^{1}{ }^{\text {, Gianandrea Salerno }}{ }^{1}$

${ }^{1}$ Department of Food and Environmental Sciences, University of Perugia, Perugia, Italy

2 Department of Entomology, Wageningen Agricultural University, Wageningen, Netherlands

3 Department of Agricultural and Forest Sciences, University of Palermo, Palermo, Italy

Corresponding Author: Francesca Frati

Email address: francescafrati@tiscali.it

Several phases of herbivorous insect attack including feeding and oviposition are known to induce plant defenses. Plants emit volatiles induced by herbivores to recruit insect parasitoids as an indirect defense strategy. So far, volatiles induced by herbivore walking and their putative role in the foraging behavior of egg parasitoids have not been investigated. In this paper we studied the response of the egg parasitoid Trissolcus basalis toward volatiles emitted by Vicia faba plants as consequence of the walking activity of the host Nezara viridula. Olfactometer bioassays were carried out to evaluate wasp responses to plants in which the abaxial or the adaxial surfaces were subjected to walking or/and oviposition. Results showed that host female walking on the abaxial but not on the adaxial surface caused a repellence effect in $T$. basalis $24 \mathrm{~h}$ after plant treatment. The emission of active volatiles also occurred when the leaf was turned upside-down, indicating a specificity of stress localization. This specificity was supported by the results, which showed that oviposition combined with feeding elicit the induction of plant volatiles, attracting the parasitoid, when the attack occurred on the abaxial surface. Analyses of plant volatile blends showed significant differences between the treatments. 
1 Submitted to: Peer J

2 Foraging behaviour of an egg parasitoid exploiting plant volatiles induced by

3 pentatomids: the role of adaxial and abaxial leaf surfaces

5 Francesca Frati ${ }^{*}$, Antonino Cusumano ${ }^{2}$, Eric Conti ${ }^{1}$, Stefano Colazza $^{3}$, Ezio Peri $^{3}$,

6 Salvatore Guarino ${ }^{3}$, Letizia Martorana ${ }^{3}$, Roberto Romani ${ }^{1}$, Gianandrea Salerno ${ }^{1}$

$7{ }^{1}$ Department of Agricultural, Food and Environmental Sciences, University of Perugia, Perugia,

8 Italy

$9 \quad{ }^{2}$ Department of Entomology, Wageningen University, Wageningen, Netherlands

$10{ }^{3}$ Department of Agricultural and Forest Sciences, University of Palermo, Palermo, Italy.

Corresponding author: francescafrati@tiscali.it 


\section{ABSTRACT}

17 Several phases of herbivorous insect attack including feeding and oviposition are known to 18 induce plant defenses. Plants emit volatiles induced by herbivores to recruit insect parasitoids as 19 an indirect defense strategy. So far, volatiles induced by herbivore walking and their putative 20 role in the foraging behavior of egg parasitoids have not been investigated. In this paper we 21 studied the response of the egg parasitoid Trissolcus basalis toward volatiles emitted by Vicia 22 faba plants as consequence of the walking activity of the host Nezara viridula. Olfactometer

23 bioassays were carried out to evaluate wasp responses to plants in which the abaxial or the 24 adaxial surfaces were subjected to walking or/and oviposition. Results showed that host female 25 walking on the abaxial but not on the adaxial surface caused a repellence effect in T. basalis $24 \mathrm{~h}$ 26 after plant treatment. The emission of active volatiles also occurred when the leaf was turned 27 upside-down, indicating a specificity of stress localization. This specificity was supported by the 28 results, which showed that oviposition combined with feeding elicit the induction of plant volatiles, attracting the parasitoid, when the attack occurred on the abaxial surface. Analyses of plant volatile blends showed significant differences between the treatments. 


\section{Introduction}

Plants belong to complex communities interacting with different organisms (Dicke et al., 2009).

In particular, plants are continuously under attack from herbivorous insects since they are used as food source, oviposition site and place to meet potential mates. After the attack, plants can activate specific responses that result in defenses against the threats (Karban \& Baldwin, 1997; Schoonhoven et al., 2005; Howe \& Jander, 2008; Schaller, 2008). These defenses can be direct, affecting negatively the physiology or behaviour of the herbivorous insect, and indirect, attracting herbivore enemies through the synthesis of volatile compounds, named herbivoreinduced plant volatiles (HIPVs) (Hare, 2011; Heil, 2014).

Plant attack by phytophagous insects can be divided into different phases acting in sequence or in concert (Hilker \& Meiners, 2010). During an attack, herbivorous insects come in contact with plants by touch or walk, and then they can feed and/or oviposit on or into the plant. Plants apparently seem passive but they are capable to sense touch by wind, vibrations (Appel \& Cocroft, 2014) and also insects (Hilker \& Meiners, 2010). In particular, they have the perception of being touched or scratched by a walking herbivore or the capacity to respond to chemical substances released from the herbivore's tarsi during the walking activity on the plant substrate (Hilker \& Meiners, 2010). During their foraging and/or oviposition activity, herbivorous insects leave their footprints on the plant substrate, which are an ensemble of tarsal pressure with surface and tarsal chemical secretions. Insect footprints have been investigated from different points of view. In the case of bumblebee, chemical footprints left on flowers are considered as an intraspecific signal used by conspecifics to avoid recently visited flowers (Eltz, 2006).

Moreover, footprints have been studied considering the role played by the pad secretions in the mechanism of insect adhesion on the surface, and the quantification of the fluid secretion rate in 
58 adhesive pads (Gorb, 2001; Dirks \& Federle, 2011). Finally, insect footprints can act as

59 interspecific signal between herbivores and parasitoids. The larval parasitoid Cotesia

60

61

62

63

64

65

66

67

68

69

70

71

72

marginiventris Cresson (Hymenoptera: Braconidae) can detect footprints left by the caterpillars of its host Spodoptera frugiperda Smith (Lepidoptera: Noctuidae) during walking activity, since the footprints are adsorbed on the plant wax surface (Rostás et al., 2008). In addition, it is known that footprints left on the substrate by true bugs have kairomonal effect for some Trissolcus and Telenomus species (Hymenoptera: Platygastridae) (Colazza et al., 1999a; Borges et al., 2003; Conti et al., 2004; Salerno et al., 2006). In particular, footprints left on the substrate by Nezara viridula (L.) (Heteroptera: Pentatomidae) females are perceived by the egg parasitoid Trissolcus basalis (Wollaston) females since they are retained onto the epicuticular waxes (Colazza et al., 2009; Lo Giudice et al., 2010; Lo Giudice et al., 2011). Moreover, the footprints left by Murgantia histrionica Hahn (Heteroptera: Pentatomidae) females on the cabbage leaves are able to induce the emission of contact synomones (Conti et al., 2010; Frati et al., 2013); in fact, $M$. histrionica footprints left on leaf surface elicit a behavioural response in the egg parasitoid Trissolcus brochymenae (Ashmead) (Conti et al., 2010). In addition, these footprint-induced synomones are adsorbed by the epicuticular waxes of cabbage leaves and subsequently exploited by the parasitoid female (Frati et al., 2013). All these examples refer to the parasitoid responses after its landing on plant. However, to date no studies have investigated whether the $N$. viridula walking activity - i.e. the ensemble of tarsal pressure with surface and the tarsal chemical secretions - is involved in eliciting the induction of specific volatile organic compounds (VOCs), which can affect the foraging behaviour of insect parasitoids. In our paper, using the tritrophic system Vicia faba (L.) - Nezara viridula - Trissolcus basalis, we tested whether herbivore walking induce the emission of plant volatiles eliciting a response of the egg parasitoid in 
81 olfactometer with particular attention on the role of leaf surface on which the biotic stress

82 occurred. Behavioural experiments are conducted to test our hypothesis and they are followed by

83 chemical analysis to confirm the recorded behavioural responses.

84 The walking activity on the substrate during the host plant exploitation represents the early phase

85 of the herbivore attack. This phase can be followed by feeding and egg deposition. Plants are

86 able to perceive herbivore feeding that is characterized by the physical damage on the plant

87 tissue (Hilker \& Meiners, 2010). However, the plant artificial wounding is not able to mimic the

88 damage inflicted by herbivore feeding that is associated to the release of herbivore regurgitants

89 into the plant wounds triggering the defensive responses (Hilker \& Meiners, 2010). Plants can

90 also sense insect oviposition (Hilker \& Meiners, 2006; Hilker \& Meiners, 2010; Hilker \&

91 Fatouros, 2015, 2016). For several systems, it is well known that insect oviposition induces the

92 emission of plant synomones that attract specific egg parasitoids (Meiners \& Hilker, 1997;

93 Meiners \& Hilker, 2000; Hilker \& Meiners, 2002; Hilker et al., 2002a; Colazza et al., 2004a,b;

94 Fatouros et al., 2005; Fatouros et al., 2007; Fatouros et al., 2008; Fatouros et al., 2009).

95 Generally, the oviposition-induced synomones are perceived by the parasitoids as olfactory

96 stimuli. However, the egg parasitoids might also respond to contact synomones perceived after

97 they have alighted on the plant (Fatouros et al., 2005; Fatouros et al., 2007; Fatouros et al.,

98 2009; Conti et al., 2010). In our study model it is clearly demonstrated in olfactometer that $T$.

99 basalis is attracted to OIPVs induced by oviposition combined with feeding of $N$. viridula

100 (Colazza et al., 2004a,b). However, the feeding damage alone does not elicit parasitoid response

101 (Colazza et al., 2004a). Phytophagous stink bug species most frequently oviposit on the

102 underside of soybean leaves (Tood \& Herezog, 1980), and this behaviour guarantees to the

103 herbivore egg masses a great protection from predators and to unfavourable microclimatic 
104 conditions (Müller \& Hilker, 2001) due for example to sunlight. This was also confirmed for $N$.

105 viridula on soybean plants where about the $80 \%$ of egg masses were laid on the abaxial leaf

106 surface (Colazza \& Bin, 1995).

107 On this account, do plants specifically respond to feeding and oviposition according to stress

108 localization? In order to answer to this question, we tested in the described system a) whether

109 there is an indirect plant response when $N$. viridula feeding damage is inflicted on adaxial leaf

110 surface and b) whether indirect plant response is stronger when the oviposition (combined with

111 feeding) occurs on abaxial part of the leaf (this situation resembles the most common case

112 occurring in nature).

113 This study could give significant information regarding the ecological consequences of

114 herbivore walking activity on plants and could contribute to understand the role of the footprints

115 as a component of the oviposition-induced plant volatile (OIPV) induction in the studied system.

116 In particular, this paper could give an important contribute in the knowledge of the interaction

117 between host and egg parasitoid mediated by OIPVs.

119 Material and Methods

120 Insects

121 The $N$. viridula colony was reared in a controlled condition chamber $\left(25 \pm 1{ }^{\circ} \mathrm{C} ; 70 \pm 10 \% \mathrm{RH}, 14\right.$

122 h:10 h L:D), inside clear plastic food containers (300 mm x $195 \mathrm{~mm}$ x $125 \mathrm{~mm}$-high) with $5 \mathrm{~cm}$

123 diameter mesh-covered holes for ventilation. Separate containers were used for nymphs and

124 adults. All stages were fed with a diet of sunflower seeds and seasonal fresh vegetables and food

125 was changed every 2-3 d. Egg masses were collected daily and used to maintain cultures of both 
$126 N$. viridula and the parasitoid T. basalis. The $N$. viridula colony was supplemented regularly

127 with field collected bugs.

128 The parasitoid T. basalis was reared on N. viridula egg masses glued on paper strips. Wasps

129 were maintained in $85 \mathrm{ml}$ glass tubes, fed with a fed with a honey-water solution and kept in 130 controlled environment room under the same rearing conditions of $N$. viridula. After emergence,

131 male and female wasps were kept together to allow mating. For all bioassays, naïve 2-4 d old

132 females were used. Females were individually isolated in small vials $1 \mathrm{hr}$ before bioassays and

133 then transferred to the bioassay room for acclimation.

\section{Plant growing}

136 Seeds of broad bean plants ( $V . f a b a \mathrm{cv}$. Superaguadulce) were immersed for $24 \mathrm{~h}$ in a slurry of 137 water and soil (1:4) to favor root nodulation. Then seeds were individually planted in plastic pots $138(9 \times 9 \times 13 \mathrm{~cm})$ filled with a mixture of agriperlite (Superlite, Gyproc Saint-Gobain, PPC Italia, 139 Italy), vermiculite (Silver, Gyproc Saint-Gobain, PPC Italia, Italy) and sand (1:1:1) and grown in 140 a climate controlled chamber $\left(24 \pm 2{ }^{\circ} \mathrm{C}, 45 \pm 10 \% \mathrm{RH}, 12 \mathrm{~h}: 12 \mathrm{~h} \mathrm{~L}: \mathrm{D}\right)$. Plants were watered daily 141 and, from one week post-germination, fertilized with an aqueous solution ( $1.4 \mathrm{~g} / \mathrm{l})$ of fertilizer

142 (5-15-45, N-P-K, Plantfol, Valagro, Italy). Fifteen days old plants with approximately four fully 143 expanded leaves were used for the experiments. Adaxial and abaxial leaf surface of Vicia faba 144 plants were considered for behavioural experiments (Fig. S1).

Plant treatments

147 Role of the herbivore walking activity in eliciting induction of plant volatiles 
148 To verify the possible volatile induction in $V$. faba plants by $N$. viridula walking activity on the 149 abaxial leaf surface, behavioral assays were carried out at different time intervals elapsing after 150 the end of the treatments $(0 \mathrm{~h}, 24 \mathrm{~h}$, and $48 \mathrm{~h})$. To obtain plants treated with only footprints 151 (tarsal pressure and tarsal chemical secretions), preventing bug feeding, gravid females with 152 excised stylets were used. For stylet excision, females were previously anaesthetized at $-4{ }^{\circ} \mathrm{C}$ for 1533 minutes inside a glass tube, in order to immobilize their labium. Afterwards, the stylets were 154 drawn from the labium with an entomological pin (no. 000), to amputate more than half their 155 length using precision micro-scissors under a stereomicroscope (Zeiss Stemi SV8) with optical 156 fiber illumination (Intralux 5000). The treated females were then placed inside a plastic dish (12 $157 \mathrm{~cm}$ diameter) allowing them to recover, and used after 1 hour to infest plants. A N. viridula 158 female with excised stylets was placed for $24 \mathrm{~h}$ on the abaxial surface of a leaf located at the second foliar stage level. The insect was set inside a small cage, made from two Petri dishes (35 $\mathrm{mm}$ diameter, $10 \mathrm{~mm}$ height) with the bottoms substituted with a fine nylon mesh and each rim of the opposite side covered with a small foam rubber ring, which was kept tightened to the leaf with the help of a clip. These treated plants were maintained at controlled conditions $\left(24 \pm 2{ }^{\circ} \mathrm{C}\right.$, $45 \pm 10 \% \mathrm{RH}, 12 \mathrm{~h}: 12 \mathrm{~h} \mathrm{~L}: \mathrm{D})$ for $24 \mathrm{~h}$ and then used for olfactometer bioassays.

To understand whether the adaxial $V$. faba leaf surface plays a different role in the volatile induction associated to herbivore walking activity, $N$. viridula females with excised stylets were individually placed for $24 \mathrm{~h}$ on the adaxial leaf surface as described above. In addition, to exclude the possible role due to the walking position of the bug females and thus the different amount of chemical tarsal secretions due to the insect pressure on the leaf surface, and to exclude the effect of the different light and temperature conditions on the two leaf surfaces,

170 plants were treated with footprints left on the abaxial leaf surface but turning the leaf upside- 
171 down. For both treatments, plants were used for olfactometer bioassays $24 \mathrm{~h}$ after the end of the 172 treatment.

173 To evaluate the role of bug gender in the induction of volatiles triggered by walking activity on

174 the abaxial leaf surface and the specificity of these volatiles, $V$. faba plants were exposed

175 respectively to the footprints left by mated $N$. viridula males and by Murgantia histrionica

176 females, both with excised stylets. Murgantia histrionica was used because it is not a host of $T$.

177 basalis (Peri et al., 2013). Considering the difference in weight between $N$. viridula (0.21 \pm 0.01

$178 \mathrm{gr})$ and $M$. histrionica $(0.087 \pm 0.001 \mathrm{gr})$ females, two bugs were used inside a clip cage during

179 each treatment. The treatments were implemented following the protocol already described and

180 the plants used for the bioassays $24 \mathrm{~h}$ after the end of the insect exposure. Healthy plants were

181 used always as control. As for treated plants, an empty clip cage was applied on the leaf of the

182 second foliar stage for $24 \mathrm{~h}$ and the plant was used $24 \mathrm{~h}$ after the clip removal. In the case of the

183 treatment represented by footprints left on abaxial upside-down turned leaf, the control was

184 obtained as described above but turning the leaf upside-down.

Role offeeding and oviposition localization in plant volatile induction

187 To elucidate whether the abaxial and adaxial $V$. faba leaf surfaces, damaged by feeding or by

188 oviposition (oviposition always combined with feeding), play a different role in volatile

189 induction according to stress localization, $N$. viridula females were individually placed for $24 \mathrm{~h}$

190 on the abaxial or on the adaxial leaf surface as described above. Plants with feeding punctures

191 plus footprints and plants with a combination of feeding punctures, a deposited egg mass and

192 footprints were used for olfactometer bioassays $24 \mathrm{~h}$ after the end of the treatment. Healthy

193 plants with an empty clip cage applied on the leaf of the second foliar stage were used as control. 


\section{Behavioural assays}

196 Wasp responses to volatile chemicals from $V$. faba plants subjected to different treatments were

197 investigated with a dual choice Y-tube olfactometer as described by Moujahed et al. (2014). The

198 different treatments were randomly assigned to each olfactometer arm at the beginning of the

199 bioassays and were reversed after testing about 10 parasitoid females. At every switch, the

200 polycarbonate olfactometer was cleaned with water and detergent and the glass parts were

201 changed with cleaned ones. At the end of the bioassays, the glass parts were then cleaned with

202 acetone and baked overnight at $180^{\circ} \mathrm{C}$.

203 Wasp females were singly introduced into the Y-tube olfactometer at the entrance of the stem

204 and allowed to move freely for $10 \mathrm{~min}$. Their behavior was recorded using a monochrome CCD

205 video camera (Sony SSC M370 CE) fitted with a 12.5-75 mm/F 1.8 zoom lens. The camera lens

206 was covered with an infrared pass filter (Kodak Wratten filter $87 \AA$ ) to remove visible

207 wavelengths. Analog video signals from the camera were digitized by a video frame grabber

208 (Canopus ${ }^{\circledR}$ ADVC 110, Grass Valley CA, USA). Digitized data were processed by XBug, a

209 video tracking and motion analysis software (Colazza et al., 1999b). Wasp response was

210 measured in terms of residence time, i.e. the time spent by the wasps in each arm during the

211 entire bioassay.

212 The Y-tube olfactometer bioassays were carried out as paired choices in which the parasitoids

213 were offered: 1) healthy plant versus plant with $N$. viridula female walking activity on abaxial

214 leaf surface at $0 \mathrm{~h}$ ( 4 couples of plants assayed with 39 parasitoids), $24 \mathrm{~h}$ ( 4 couples of plants

215 assayed with 41 parasitoids) and $48 \mathrm{~h}$ ( 4 couples of plants assayed with 41 parasitoids) from the

216 end of the treatment; 2) healthy plant versus plant with $N$. viridula female walking activity on 
217 adaxial leaf surface (4 couples of plants assayed with 40 parasitoids); 3 ) healthy plant with

218 upside-down abaxial leaf versus plant with $N$. viridula female walking activity on upside-down

219 abaxial leaf (5 couples of plants assayed with 40 parasitoids); 4) healthy plant versus plant with

$220 N$. viridula walking activity of male on abaxial leaf surface (4 couples of plants assayed with 34

221 parasitoids); 5) healthy plant versus plant with $M$. histrionica females walking activity on

222 abaxial leaf surface (4 couples of plants assayed with 39 parasitoids); 6) healthy plant versus

223 plant with $N$. viridula female walking activity associated with feeding punctures on abaxial (5

224 couples of plants assayed with 44 parasitoids) or on adaxial leaf surface (4 couples of plants

225 assayed with 34 parasitoids); 7) healthy plant versus plant with $N$. viridula female walking

226 activity associated with feeding punctures and oviposition on abaxial (6 couples of plants

227 assayed with 44 parasitoids) or on adaxial leaf surface (6 couples of plants assayed with 58

228 parasitoids).

229 All bioassays were conducted from $\sim 09: 00 \mathrm{~h}$ to 13:00 h under controlled conditions $\left(26 \pm 1^{\circ} \mathrm{C}\right.$, $23050 \pm 5 \% \mathrm{RH})$.

231

232 Collection and analysis of VOCs

233 Cylindrical glass chambers (inner $\varnothing=10 \mathrm{~cm}, \mathrm{~h}=30 \mathrm{~cm}$ ), with a two semi-circular-part joint

234 base made by Teflon with a 2-cm hole in the center to permit the insertion of the plant at the

235 level of collar were used to collect headspace volatiles only from the epigeous part of the plant.

236 Teflon taping was placed around the plant collar to make it tight on the Teflon base. Before each

237 collection, the glass chamber and Teflon base were washed with water and detergent, rinsed with

238 acetone, and baked overnight at $120^{\circ} \mathrm{C}$. A whole plant was inserted in the chamber and flushed 
239 with active charcoal filtered air at flux rate of $300 \mathrm{ml} \mathrm{min}^{-1}$ for $3 \mathrm{~h}$ using a pump NMP 830

240 KNDC 12V (KNF, Milano, Italy).

241 VOC emissions by plants were collected using adsorbent traps, placed at the chamber outlet,

242 made by glass tubes filled with PorapakQ (SigmaAldrich; $60 \mathrm{mg}$, 80-100 mesh), which were

243 pre-cleaned with hexane and then heat conditioned for at least $2 \mathrm{~h}$ in a stream of nitrogen (100

$244 \mathrm{ml} / \mathrm{min}$ ) at $130^{\circ} \mathrm{C}$. After $3 \mathrm{~h}$ traps were eluted with $700 \mu \mathrm{l}$ of hexane, and the resulting extracts

245 were stored at $-20^{\circ} \mathrm{C}$ in glass vials with Teflon cap liners until used for gas chromatography

246 (GC) analysis. On the basis of behavioral results only the treatments significantly affecting the

247 parasitoid response were used for volatile collection. In particular, VOCs were collected from

248 healthy plants as control, plants with $N$. viridula female walking activity on abaxial leaf surface

249 at $24 \mathrm{~h}$, plants with $N$. viridula female walking activity on adaxial leaf surface; plants with $N$.

250 viridula female walking activity + feeding punctures + oviposition on abaxial leaf surface and

251 plants with $N$. viridula female walking activity + feeding punctures + oviposition on adaxial leaf

252 surface. For each treatment, 6 plants were sampled. Blank measurements were carried out before

253 every set of measurements, by sampling air from the chamber, in this case the Teflon base was

254 tap using Teflon.

255 Gas chromatography-mass spectrometry (GC-MS) analysis was performed on a Hewlett-Packard

2565890 GC system interfaced with an HP5973 quadruple mass spectrometer. For each sample, $1 \mu \mathrm{l}$

257 of extract was injected onto a HP5-MS column (5\% diphenyl-95\%dimethyl polysiloxane 30m $\times$

$2580.2 \mathrm{~mm}, 0.25-\mu \mathrm{m}$ film, J \& W Scientific, Folsom CA, USA) in splitless mode. Injector and

259 detector temperatures were $260^{\circ} \mathrm{C}$ and $280^{\circ} \mathrm{C}$ respectively. Helium was used as the carrier gas.

260 The $\mathrm{GC}$ oven temperature program was $40{ }^{\circ} \mathrm{C}$ for $5 \mathrm{~min}$, then, increased by $10^{\circ} \mathrm{C} / \mathrm{min}$ to $250{ }^{\circ} \mathrm{C}$. 
261 Electron impact ionization spectra were obtained at $70 \mathrm{eV}$, recording mass spectra from 40 to $262550 \mathrm{amu}$.

263 Peak area of each detected compound was calculated. Compounds were tentatively identified,

264 based on comparison of RI and mass spectra with those in Adams (2007),

265 http://www.pherobase.com and the NIST 1998 libraries. For (E)-2-Hexenal, (Z)-3-Hexenyl

266 acetate, Benzaldehyde, $\beta$-Ocimene, $\alpha$-Pinene, $\alpha$-Myrcene, Linalool, $\beta$-Caryophyllene, Octanal,

267 Nonanal, Decanal, Octan-1-ol, Tetradecane and Isomenthone, tentative identifications by GC-

268 MS were confirmed by injection of authentic standards. Standars used were obtained from

269 Sigma-Aldrich (Germany).

270

271 Statistical analysis

272 Data were analyzed by linear mixed model (LMM) with the plant treatment as fixed effect and

273 parasitoid nested within each plant pairs as random effects to account for pseudoreplication.

274 Significance of the fixed term in the model was determined using likelihood ratio tests (LRTs)

275 comparing the model with and without the factor in question (Crawley, 2007). Because this

276 approach compares models with different fixed effect structures, Maximum Likelihood (ML)

277 was specified in the models instead of Restricted Maximum Likelihood (REML) (Crawley,

278 2007). Model fit was assessed with residual plots. All statistical analyses were carried out with R

279 software, version 3.1.3 (R Core Team, 2015).

280 Data from analysis of volatiles were analyzed by multivariate analysis using projection to latent

281 structures discriminant analysis (PLS-DA) with SIMCA-P+ 12.0 software program (Umetrics

282 AB, Umeå, Sweden). The projection method determines if samples belonging to the different 
283 treatment groups can be separated on the basis of quantitative and qualitative differences in their

284 volatile blends.

285

\section{Results}

287 Role of the herbivore walking activity in eliciting induction of plant volatiles

288 Trissolcus basalis females were able to discriminate between $V$. faba healthy plants and plants

289 with $N$. viridula walking activity on abaxial leaf surface but not with $N$. viridula walking activity

290 on adaxial leaf surface.

291 In particular, T. basalis females significantly preferred volatiles emitted by healthy plants as

292 control compared to plants with $N$. viridula walking activity on abaxial leaf surface, assayed 24

$293 \mathrm{~h}$ after the end of the treatment $\left(\chi^{2}=19.016 ; \mathrm{df}=1 ; P<0.0001\right)$. Whereas differences were not

294 significant when $0 \mathrm{~h}\left(\chi^{2}=0.645 ; \mathrm{df}=1 ; P=0.422\right)$ and $48 \mathrm{~h}\left(\chi^{2}=0.117 ; \mathrm{df}=1 ; P=0.733\right)$ were

295 considered (Fig. 1 A).

296 Wasps showed a significant preference for volatiles emitted by the control compared to those

297 released by plants with $N$. viridula walking activity on abaxial leaf turned upside-down

$298\left(\chi^{2}=11.162 ; \mathrm{df}=1 ; P=0.0008\right)$ (Fig. $\left.1 \mathrm{~B}\right)$. When comparing plants with $N$. viridula walking

299 activity on adaxial leaf surface and healthy plants, no significant differences were displayed

300 between test and control $\left(\chi^{2}=0.260 ; \mathrm{df}=1 ; P=0.611\right)$ (Fig. $\left.1 \mathrm{~B}\right)$.

301 The walking activity of $N$. viridula male $\left(\chi^{2}=0.56 ; \mathrm{df}=1 ; P=0.454\right)$ or that of $M$. histrionica

302 females on abaxial leaf surface $\left(\chi^{2}=2.108 ; \mathrm{df}=1 ; P=0.147\right)$ did not stimulate a significant

303 response from wasps compared to healthy plants (Fig. $1 \mathrm{C}$ ).

304

305

Role offeeding and oviposition localization in plant volatile induction 
306 Nezara viridula oviposition, combined with feeding and footprints, on plant surface induces

307 OIPVs triggering the parasitoid response only when egg deposition occurs on abaxial leaf

308 surface. In particular, parasitoid females showed a significant preference for volatiles released by

309 plants exposed to $N$. viridula walking, feeding and oviposition activities on abaxial leaf surface

$310\left(\chi^{2}=7.331 ; \mathrm{df}=1 ; P=0.007\right)$ compared to the control (Fig. 2), whilst, when the treatment was

311 located on adaxial leaf surface no significant choice was displayed $\left(\chi^{2}=0.247 ; \mathrm{df}=1 ; P=0.620\right)$

312 (Fig. 2). In the case of $V$. faba plants with $N$. viridula walking and feeding activities on abaxial

$313\left(\chi^{2}=0.164 ; \mathrm{df}=1 ; P=0.685\right)$ or adaxial leaf surface $\left(\chi^{2}=0.392 ; \mathrm{df}=1 ; P=0.531\right)$, no preference for

314 test and control was shown (Fig. 2).

Plant VOC analysis

317 The following 23 compounds were detected in $V$. faba plants differently treated: $(E)$-2-Hexenyl

318 butyrate, Isomenthone, Hexyl butyrate, Undecan-2-one, $\alpha$-Myrcene, unknown 1, Ethylbenzene,

$319 \beta$-Caryophyllene, $\alpha$-Pinene, Hexan-1-ol, unknown 2, Octanal, Benzaldehyde, (Z)-3-hexen-1-ol,

320 (E)-2-Hexenal, Tetradecane, $(E)-\beta$ Ocimene, $(Z)$-3-hexenyl acetate, Octan-1-ol, Decanal,

321 Linalool, 6-Methyl-5-hepten-2-one, nonanal, 1-Octen-3-ol. The PLS-DA comparison including

322 samples of all treatments, resulted in a model with one significant principal component (PC1;

$323 \mathrm{R} 2 \mathrm{X}=0.175 ; \mathrm{R} 2 \mathrm{Y}=0.148 ; \mathrm{Q} 2=0.055 ;$ Fig. 3). The model separated healthy plants and plants

324 with $N$. viridula walking on adaxial leaf surface from plants with $N$. viridula walking on abaxial

325 leaf surface and plants with $N$. viridula walking associated with feeding punctures and

326 oviposition on abaxial or adaxial leaf surface. In particular, the volatile blend emitted by plants

327 with footprints left on abaxial leaf surface is different from that emitted by plants with footprints

328 left on adaxial leaf surface and from healthy plants. Examination of the loading plot showed that 
329 a group of 8 compounds contributed the most to explaining the variation in the model (Fig. 3B).

330 These compounds have the following retention time (min) and corresponding VIP values

331 (variable importance for the projection): $(E)-2$-Hexenyl butyrate $=1.54$; Isomenthone $=1.48$;

332 Hexyl butyrate $=1.31 ;$ Undecan-2-one $=1.28 ; \alpha$-Myrcene $=1.21 ;$ Unknown $=1.10$;

333 Ethylbenzene $=1.10 ; \beta$-Caryophyllene $=1.05$.

\section{Discussion}

Our study suggests that the herbivore walking activity on the substrate elicits plant volatile induction. The egg parasitoid T. basalis was able to discriminate between volatiles from healthy plants and volatiles from plants with footprints of $N$. viridula females. However, the parasitoid response was time interval dependent, as it was recorded after $24 \mathrm{~h}$ from the treatment, while no response was shown at the time intervals of 0 and $48 \mathrm{~h}$ after the end of bug walking activity. The leaf surface is actively involved in this induction. In fact, only when the herbivore walking activity occurred on the abaxial leaf surface the parasitoid response was shown. This is demonstrated by the fact that turning the leaf upside-down and treating the abaxial leaf surface the parasitoid response did not change. In addition, there were no behavioral responses by the parasitoid when leaf treatment occurred with walking activity of $N$. viridula males and of the non-associated host $M$. histrionica females. The combination of walking and feeding activities on adaxial and abaxial leaf surface did not induce wasp behavioral responses. Furthermore, our research reveals that $N$. viridula oviposition (combined with walking and feeding) elicited the induction of OIPVs when it has occurred on abaxial leaf surface. This result confirms the induction of OIPVs in $V$. faba by $N$. viridula, already reported in previous papers where the

351 oviposition was generally recorded on the abaxial leaf surface, as happens in natural conditions, 
352 because the plant exposition was carried out in a wood-framed, nylon mesh cage (Colazza et al.,

353 2004a) or in a net bag (Moujahed et al., 2014) with $N$. viridula females free to explore the whole

354 plant.

355 It is possible to hypothesize that, in our system, the walking activity of $N$. viridula female on

356 abaxial $V$. faba leaf surface elicited the emission of putative induced plant volatiles. The

357 behavioural data seem to be supported by volatile analyses since the PLS-DA showed that the

358 whole blend of plant volatiles changes according to stress localization, i.e. when footprints are

359 left on the abaxial or adaxial leaf surface. An effect on wasp behaviour of volatilization of

360 precursors deposited by the walking insects on leaf surface could be excluded considering that

361 the wasp response was recorded only when the abaxial leaf surface, but not the adaxial leaf

362 surface, is contaminated. Although the induced volatiles associated with the herbivore walking

363 activity did not attract the parasitoid but favoured a repellence effect, they actually might act

364 similarly to a synomone. In fact, our hypothesis is that these induced volatiles could give the

365 parasitoid information that the plant has been visited by the herbivore but it is not there anymore.

366 This repellence for 'old' $(24 \mathrm{~h})$ footprint is not showed for fresh footprints $(0 \mathrm{~h})$ as they could

367 indicate the possibility of herbivores around. Considering that the parasitoids are under selection

368 pressure to maximize their foraging efficiency in order to improve their ecological fitness

369 (Tamiru et al., 2015), it is fundamental that they should not waste time exploiting these chemical

370 cues, which may be unreliable indicators of the egg presence. The emission of putative induced

371 volatiles associated with herbivore walking activity is not due to the simple contact with the

372 herbivorous insect but probably to the secretions released at tarsal level and probably produced

373 by tibial glands (Romani, pers. comm). These secretions, which concur to the herbivore adhesion

374 on the leaf surface, interact with the plant in a specific manner depending on whether the 
375 interaction occurs with the abaxial or the adaxial leaf surface. In addition, the adhesion of the $N$.

376 viridula tarsi on the substrate, and in particular of the claws during the stationary contact on the

377 surface, does not provoke evident mechanical damage on both leaf surfaces as morphological

378 investigations by scanning electron microscope reveal no differences between healthy and

379 footprint-treated $V$. faba leaf surfaces (Romani, pers. comm.). Our results clearly show that the

380 two leaf surfaces interact differently with $N$. viridula walking activity. This phenomenon could

381 be explained considering the following morphological, anatomical and structural differences

382 between abaxial and adaxial leaf surfaces. The wax composition of the abaxial leaf surface may

383 differ from that of the adaxial surface (Müller \& Hilker, 2001); leaf palisade mesophyll cells are

384 beneath the adaxial leaf surface and spongy mesophyll in the lower half; stomatal density is

385 higher on the abaxial surface respect to adaxial surface of leaves (Willmer \& Fricker, 1996a);

386 abaxial guard cells are typically larger and stomatal pores are wider under conditions favouring

387 opening (Willmer \& Fricker, 1996b); finally, gas exchange between a leaf or leaflet and the 388 atmosphere occurs mainly via abaxial stomata (Lu, 1988). In the case of $V$. faba, leaves have

389 stomata on both abaxial and adaxial epidermis, but the number of stomata per $\mathrm{mm}^{2}$, the stomata

390 width and length on the abaxial epidermis of the leaflet is higher than on the adaxial epidermis

391 (Pekşen et al., 2006). The pad secretions of $N$. viridula are released during walking on the

392 substrate (Salerno, pers. comm.) but how they interact with leaf surface or if /how they move

393 into the leaf remains unknown. Moreover, the exposure to light and/or temperature of the tarsal

394 secretions could vary from abaxial and adaxial leaf surface, but in our bioassays we can exclude

395 the effect of the different light and temperature conditions on the two leaf surfaces since no

396 differences were recorded in the T. basalis responses towards plants with the abaxial leaf surface

397 with $N$. viridula walking and plants with the upside-down turned leaf. 
398 The two leaf surfaces interact differently not only with $N$. viridula walking but also with the

399 eggs laid by the herbivore. It is known that plants react to herbivore oviposition activating 400 defensive responses. In particular egg deposition, also associated with wounding, induces the 401 emission of volatiles attracting antagonists of the herbivores. The majority of herbivore insects 402 oviposit on plant leaves (Hilker \& Meiners, 2011) preferring the abaxial leaf surface rather than 403 the adaxial (Müller \& Hilker, 2001). Oviposition on adaxial leaf surface exposes eggs to 404 sunlight, to unfavourable microclimatic conditions (Willmer, 1986) and to egg predators and 405 parasitoids (Müller \& Hilker, 2001). As recorded for other stink bug species (Tood \& Herzog, 406 1980), N. viridula tends to oviposit on the abaxial leaf surface (Colazza \& Bin, 1995). Our data 407 show that $N$. viridula oviposition (combined with feeding and footprints) on plant surface 408 induces OIPVs triggering the parasitoid response only when egg deposition occurs on abaxial 409 leaf surface. However, these results are not explained by the PLS-DA, as the model does not 410 separate between volatiles emitted in response to oviposition occurring on the abaxial or adaxial 411 leaf surfaces. However, in our analysis $(E)$ - $\beta$-caryophyllene appears to be a VIP compound 412 confirming its potential role as synomone for T. basalis as previously hypothesized by Colazza 413 et al. (2004b).

414 PLS-DA analyses take into account all volatiles but the wasps likely use a specific subset of 415 compounds of the total blend (Clavijo McCormick et al., 2012). Thus, a mismatch between 416 behavioral responses and chemical analyses could be due to the fact that the parasitoids focused 417 on some key volatiles associated with $N$. viridula-egg deposition on the abaxial leaf surface 418 which constitute the active blend, whereas PLS-DA takes the whole blend into account.

419 In conclusion, our results confirm that the signals mediating the interaction between plants, 420 herbivores and parasitoids are effective and finely tuned since they guarantee maximization of 
421 the chance to find the suitable host. In particular, data reported in this paper elucidate, first, the

422 role of the herbivore walking activity in a simplified experimental set up (walking alone, without

423 feeding and oviposition, only occasionally occur in nature), and second, the key role of the

424 abaxial leaf surface in mediating the volatile communication between T. basalis and its host.

425

426

427

428

429

430

431

432

433

434

435

436

437

438

439

440

441

442

\section{Acknowledgements}

We are grateful to Andrea Luchetti for rearing the insects and to Daniela Fortini and Cesare Dentini for growing Vicia faba plants. We thank Anna Laureti for helping in data collection.

\section{References}

Adams RP. 2007. Identification of essential oil components by gas chromatography/mass spectrometry, 4th edn. Allured Publishing Corporation, Carol Stream.

Appel HM, Cocroft RB. 2014. Plants respond to leaf vibrations caused by insect herbivore Chewing. Oecologia 175:1257-1266. DOI 10.1007/s00442-014-2995-6.

Borges M, Colazza S, Ramirez-Lucas P, Chauhan KR, Blassioli Moraes MC, Aldrich JR. 2003. Kairomonal effect of walking traces from Euschistus heros (Heteroptera: Pentatomidae) on two strains of Telenomus podisi (Hymenoptera: Scelionidade). Physiological Entomology 28:349_ 355.

Clavijo McCormick A, Unsicker SB, Gershenzon J. 2012. The specificity of herbivore-induced plant volatiles in attracting herbivore enemies. Trends in Plant Science 17:303-310. 
443 Colazza S, Bin F. 1995. Efficiency of Trissolcus basalis (Hymenoptera: Scelionidae) as an Egg

444 Parasitoid of Nezara viridula (Heteroptera: Pentatomidae) in Central Italy. Environmental

445 Entomology 24:1703-1707.

446 Colazza S, Salerno G, Wajnberg E. 1999a. Volatile and contact chemicals released by Nezara

447 viridula (Heteroptera: Pentatomidae) have a kairomonal effect on the egg parasitoid Trissolcus

448 basalis (Hymenoptera: Scelionidae). Biological Control 16:310-317.

449 Colazza S, Peri D, Salerno G, Peri E, Lo Pinto M, Liotta G. 1999b. Xbug, a video tracking and 450 motion analysis system for LINUX. XII International Entomophagous Insects Workshop. Pacific

451 Grove, California, September 26-30.

452 Colazza S, Fucarino A, Peri E, Salerno G, Conti E, Bin F. 2004a. Insect oviposition induces

453 volatiles emission in herbaceous plant that attracts egg parasitoids. Journal of Experimental

454 Biology 207:47-53.

455 Colazza S, McElfresh JS, Millar JG. 2004b. Identification of volatile synomones, induced by

456 Nezara viridula feeding and oviposition on bean spp., that attract the egg parasitoid Trissolcus

457 basalis. Journal of Chemical Ecology 30:945-964.

458 Colazza S, Lo Bue M, Lo Giudice D, Peri E. 2009. The response of Trissolcus basalis to 459 footprint contact kairomones from Nezara viridula females is mediated by leaf epicuticular 460 waxes. Naturwissenschaften 96:975-981.

461 Conti E, Salerno G, Bin F, Vinson SB. 2004. The role of host semiochemicals in parasitoid 462 specificity: a case study with Trissolcus brochymenae and Trissolcus simoni on pentatomid 463 bugs. Biological Control 29:435-444. 
464 Conti E, Salerno G, Leombruni B, Frati F, Bin F. 2010. Short-range allelochemicals from a

465 plant-herbivore association: a singular case of oviposition-induced synomone for an egg

466 parasitoid. Journal of Experimental Biology 213:3911-3919.

467 Crawley NJ. 2007. The R book. Chichester England: Wiley J. \& Sons.

468 Dicke M, van Loon JJA, Soler R. 2009 Chemical complexity of volatiles from plants induced by 469 multiple attack. Nature Chemical Biology 5:317-324.

470 Dirks JH, Federle W. 2011. Mechanisms of fluid production in smooth adhesive pads of insects.

471 Journal of the Royal Society Interface 8:952-960.

472 Eltz T. 2006. Tracing pollinator footprints on natural flowers. Journal of Chemical Ecology $473 \quad 32: 907-915$.

474 Fatouros NE, Bukovinszkine'Kiss G, Kalkers LA, Soler Gamborena R, Dicke M, Hilker M. 475 2005. Oviposition-induced plant cues: do they arrest Trichogramma wasps during host location?

476 Entomologia Experimentalis et Applicata 115:207-215.

477 Fatouros NE, Bukovinszkine'Kiss G, Dicke M, Hilker M. 2007. The response specificity of 478 Trichogramma egg parasitoids towards infochemicals during host location. Journal of Insect 479 Behavior 20:53-65.

Fatouros NE, Dicke M, Mumm R, Meiners T, Hilker M. 2008. Foraging behavior of egg parasitoids exploiting chemical information. Behavioral Ecology 19:677-689.

482 Fatouros NE, Pashalidou FG, Aponte Cordero WV, van Loon JJA, Mumm R, Dicke M, Hilker 483 M, Huigens ME. 2009. Anti-aphrodisiac compounds of male butterflies increase the risk of egg 484 parasitoid attack by inducing plant synomone production. Journal of Chemical Ecology 35:13734851381. 
486 Frati F, Salerno G, Conti E. 2013. Cabbage waxes affect Trissolcus brochymenae response to

487 short-range synomones. Insect Science 20:753-762

488 Gorb SN. 2001. Attachment devices of insect cuticle. Dordrecht, The Netherlands: Kluwer 489 Academic.

490 Hare JD. 2011. Ecological role of volatiles produced by plants in response to damage by 491 herbivorous insects. Annual Review of Entomology 56:161-80.

492 Heil M. 2014. Herbivore-induced plant volatiles: targets, perception and unanswered questions.

493 New Phytologist 204:297-306.

494 Hilker M, Fatouros NE. 2015. Plant responses to insect egg deposition. Annual Review of 495 Entomology 60:493-515.

496 Hilker M, Fatouros NE. 2016. Resisting the onset of herbivore attack: plants perceive and 497 respond to insect eggs. Current Opinion in Plant Biology. 32:9

498 Hilker M, Meiners T. 2002. Induction of plant responses to oviposition and feeding by

499 herbivorous arthropods: a comparison. Entomolologia Experimentalis et Applicata 104:181-192.

500 Hilker M, Rohfritsch O, Meiners T. 2002a. The plant's response towards insect egg deposition.

501 In: Hilker M \& Meiners T, eds. Chemoecology of Insect Eggs and Egg Deposition. Berlin:

502 Blackwell Publishers, 205-233.

503 Hilker M, Meiners T. 2006. Early herbivore alert: Insect eggs induce plant defense. Journal of

504 Chemical Ecology 32:1379-1397.

505 Hilker M, Meiners T. 2010. How do plants "notice" attack by herbivorous arthropods?

506 Biological Reviews 85:267-280. 
507 Hilker M, Meiners T. 2011. Plants and insect eggs: how do they affect each other?

508 Phytochemistry, 72:1612-1623.

509 Howe GA, Jander G. 2008. Plant immunity to insect herbivores. Annual Review of Plant

510 Biology 59:41-66.

511 Karban R, Baldwin IT. 1997. Induced responses to herbivory. Chicago: Chicago University

512 Press.

513 Lo Giudice D, Peri E, Lo Bue M, Colazza S. 2010. Plant surface of vegetable crops mediate

514 interactions between chemical footprints of true bugs and their egg parasitoids. Communicative 515 and Integrative Biology 1:70-74.

516 Lo Giudice D, Riedel M, Rostás M, Peri E, Colazza S. 2011. Host sex discrimination by an egg 517 parasitoid on Brassica leaves. Journal of Chemical Ecology 37:622-628.

518 Lu Z. 1988. The sensitivity of adaxial and abaxial stomatal resistance in wheat leaf to soil water 519 stress. Acta Phytophysiologica Sinica 14:223-227.

520 Meiners T; Hilker M. 1997. Host location in Oomyzus gallerucae (Hymenoptera: Eulophidae), 521 an egg parasitoid of the elm leaf beetle Xanthogaleruca luteola (Coleoptera: Chrysomelidae).

522 Oecologia 112:87-93.

523 Meiners T, Hilker M. 2000. Induction of plant synomones by oviposition of a phytophagous

524 insect. Journal of Chemical Ecology 26:221-232.

525 Moujahed R, Frati F, Cusumano A, Salerno G, Conti E, Peri E, Colazza S. 2014. Egg parasitoid 526 attraction toward induced plant volatiles is disrupted by a non-host herbivore attacking above or 527 below ground plant organs. Frontiers in plant science 5, 10.3389/fpls.2014.00601 
528 Müller C, Hilker M. 2001. Host finding and oviposition behavior in a chrysomelid specialist-

529 the importance of host plant surface waxes. Journal of Chemical Ecology 27:985-994.

530 Pekşen E, Pekşen A, Artik C. 2006. Comparison of leaf and stomatal characteristic of Faba bean

531 (Vicia faba L.). Journal of Biological Science 6:360-364.

532 Peri E, Frati F, Salerno G, Conti E, Colazza S. 2013. Host Chemical Footprints Induce Host Sex

533 Discrimination Ability in Egg Parasitoids. Plos One 8:e79054

534 R Core Team 2015 R: A language and environment for statistical computing. Vienna (Austria).

535 R Foundation for statistical computing.

536 Rostás M, Ruf D, Zabka V., Hildebrandt U. 2008. Plant surface wax affects parasitoid’s

537 response to host footprints. Naturwissenschaften 95:997-1002.

538 Salerno G, Conti E, Peri E, Colazza S, Bin F. 2006. Kairomone involvement in the host

539 specificity of the egg parasitoid Trissolcus basalis (Hymenoptera: Scelionidae). European

540 Journal of Entomology 103:311-318.

541 Schaller A. 2008. Induced plant resistance to herbivory. Springer.

542 Schoonhoven LM, van Loon JJA, Dicke M. 2005. Insect-plant biology. Oxford: Oxford

543 University Press.

544 Tamiru A, Zeyaur RK, Bruce TJA. 2015. New directions for improving crop resistance to insects

545 by breeding for egg induced defence. Current Opinion in Insect Science 9:51-55.

546 Tood JW, Herzog DC. 1980. Sampling phytophagous pentatomidae on soybean. In: Kogan M \&

547 Herzog DC, eds. Sampling methods in soybean entomology. New York Inc.: Springer-Verlag. 
548 Willmer C, Fricker M. 1996a. The distribution of stomata. In: Willmer C \& Fricker M, eds.

549 Stomata. London: Chapman \& Hall, 18-19.

550 Willmer C, Fricker M. 1996b. Stomatal responses to environmental factors. In: Willmer C \&

551 Fricker M, eds. Stomata. London: Chapman \& Hall, 126-191.

552 Willmer P. 1986. Microclimatic effects on insects at the plant surface. In: Juniper B \&

553 Southwood TRE, eds. Insects and the plant surface.London: Arnold, 65-80. 


\section{FIGURES}

\section{Figure 1. Possible role of bug walking activity in plant volatile induction.}

557 Response of T. basalis females in a Y-tube olfactometer to volatiles from $V$. faba plants treated:

558 A) with $N$. viridula female walking activity on the abaxial leaf surface, and assayed $0 \mathrm{~h}, 24 \mathrm{~h}$, 559 and $48 \mathrm{~h}$ after the treatment versus healthy plants; B) with $N$. viridula female walking activity on 560 overturned leaf versus healthy plants with overturned leaf and plants with $N$. viridula female 561 walking activity on adaxial leaf surface versus healthy plants; C) with $M$. histrionica (non562 associated host) walking activity of 2 females on abaxial leaf surface versus healthy plants and 563 treated with $N$. viridula walking activity of male on abaxial leaf surface versus healthy plants.

564 Bars represent mean $( \pm$ SEM) of the time spent by wasp females in each arm over an observation 565 period of $600 \mathrm{~s}$. Asterisks $(* * *)$ indicate $\mathrm{p}<0.001$ by linear mixed model LMM. ns $=$ not 566 significant. $\mathrm{n}=$ number of replicates 


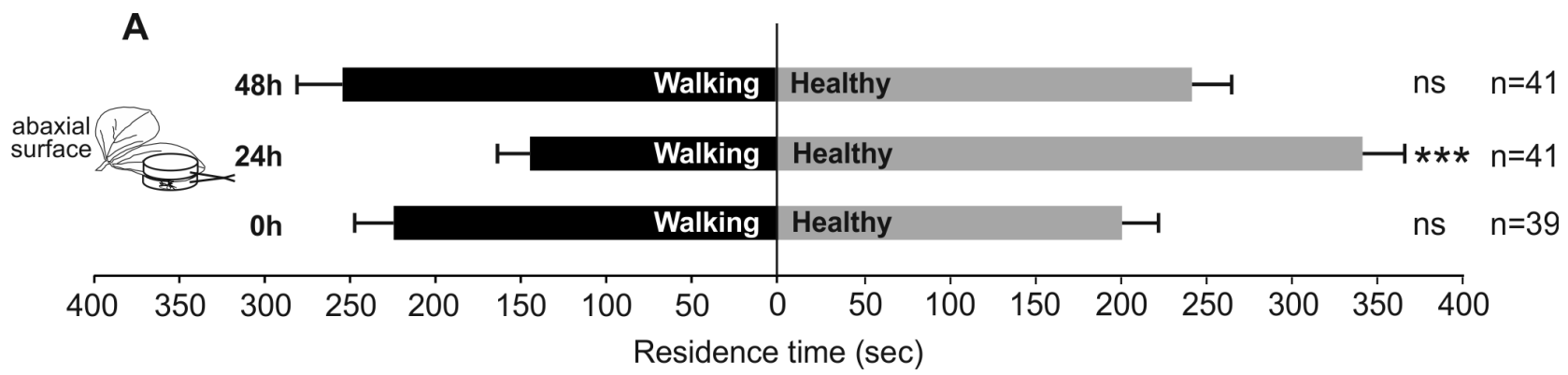

B
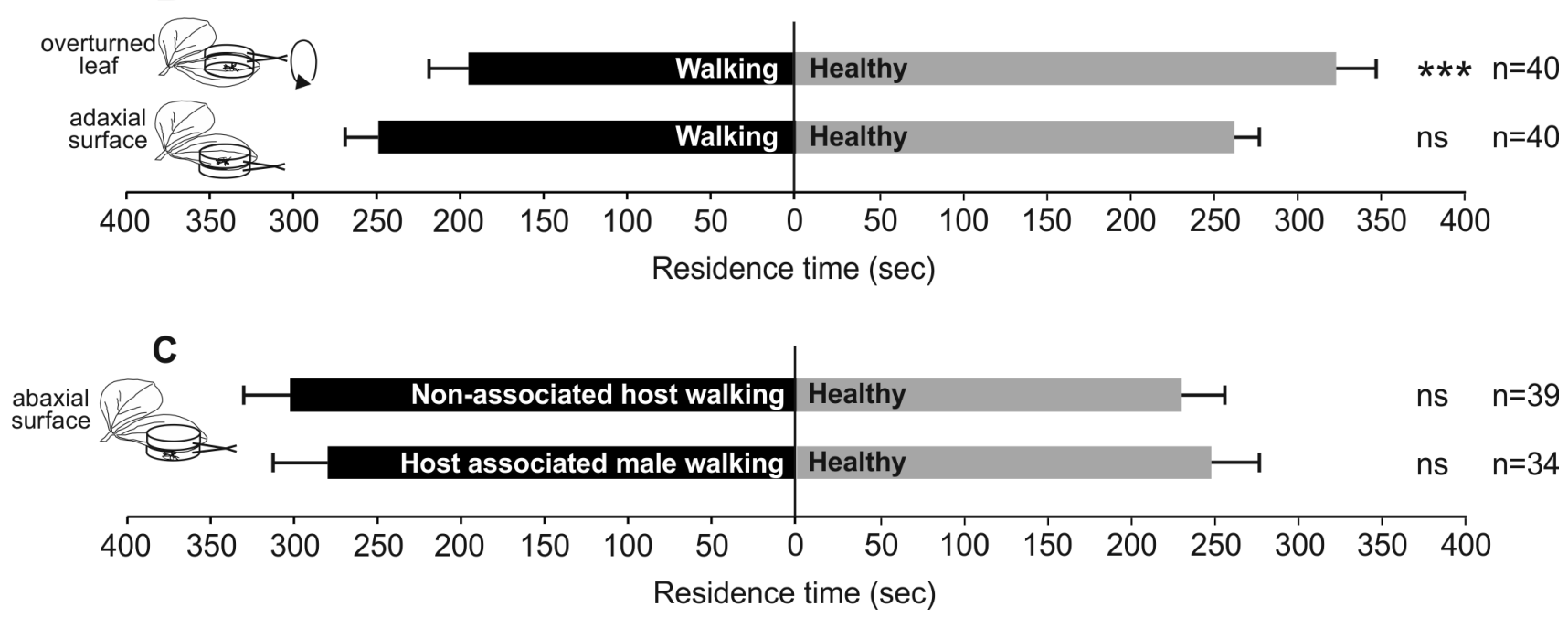
570 Figure 2. Role of stress localization in the induction of plant volatiles. Response of T. basalis

571 females in a Y-tube olfactometer to volatiles emitted by 1) $V$. faba plants with $N$. viridula female

572 walking activity associated with feeding punctures and oviposition on the adaxial leaf surface

573 versus healthy plants; 2) plants with $N$. viridula female walking activity associated with feeding

574 punctures on the adaxial leaf surface versus healthy plants; 3) plants with $N$. viridula female

575 walking activity associated with feeding punctures and oviposition on the abaxial leaf surface

576 versus healthy plants and 4) plants with $N$. viridula female walking activity associated with

577 feeding punctures left on the abaxial leaf surface versus healthy plants. Bars represent mean $( \pm$

578 SEM) of the time spent by wasp females in each arm over an observation period of 600s.

579 Asterisks $(* *)$ indicate $\mathrm{p}<0.01$ by linear mixed model LMM. $\mathrm{ns}=$ not significant. $\mathrm{n}=$ number of

580 replicates

581

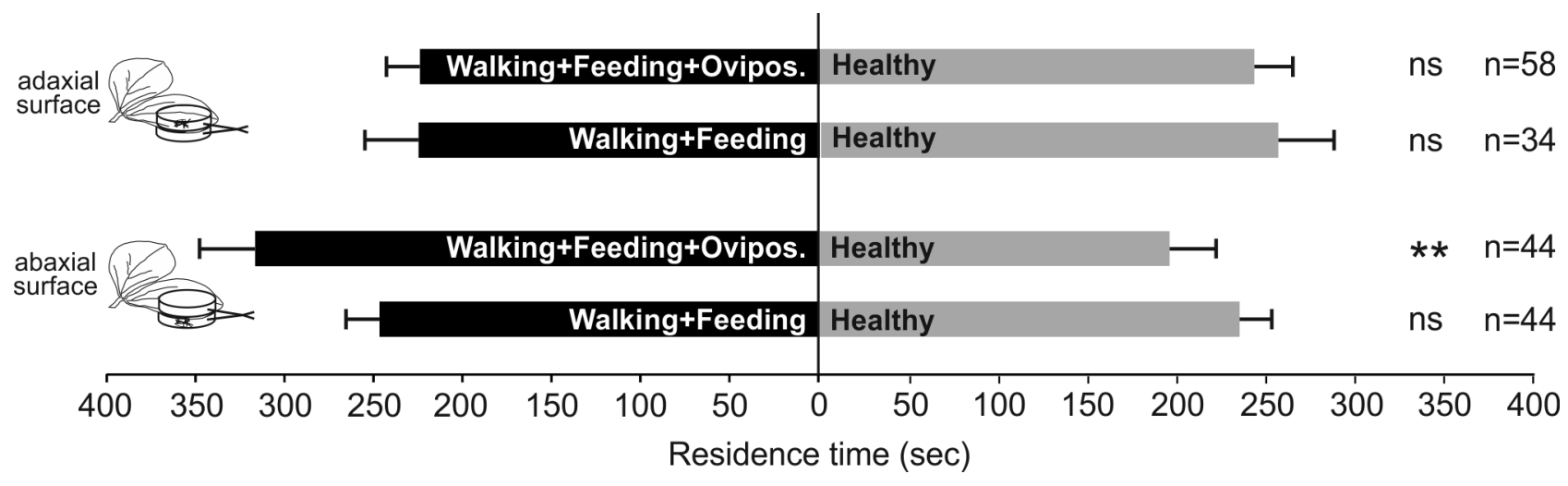


584 Figure 3. Projection to latent structures discriminant analysis (PLS-DA) comparison of the 585 volatile compounds emitted by individual $\boldsymbol{V}$. faba plants. (A) Score plot of the samples, with 586 the percentage of explained variation in parentheses. The PLS-DA resulted in a model with one 587 significant principal components (PCs). The ellipse defines the Hotelling'sT2 confidence region 588 (95\%). (B) Loading plot of the first two components of the PLS-DA, showing the contribution of 589 each of the compounds toward the model. Numbers refer to the retention time of volatile 590 compounds. 


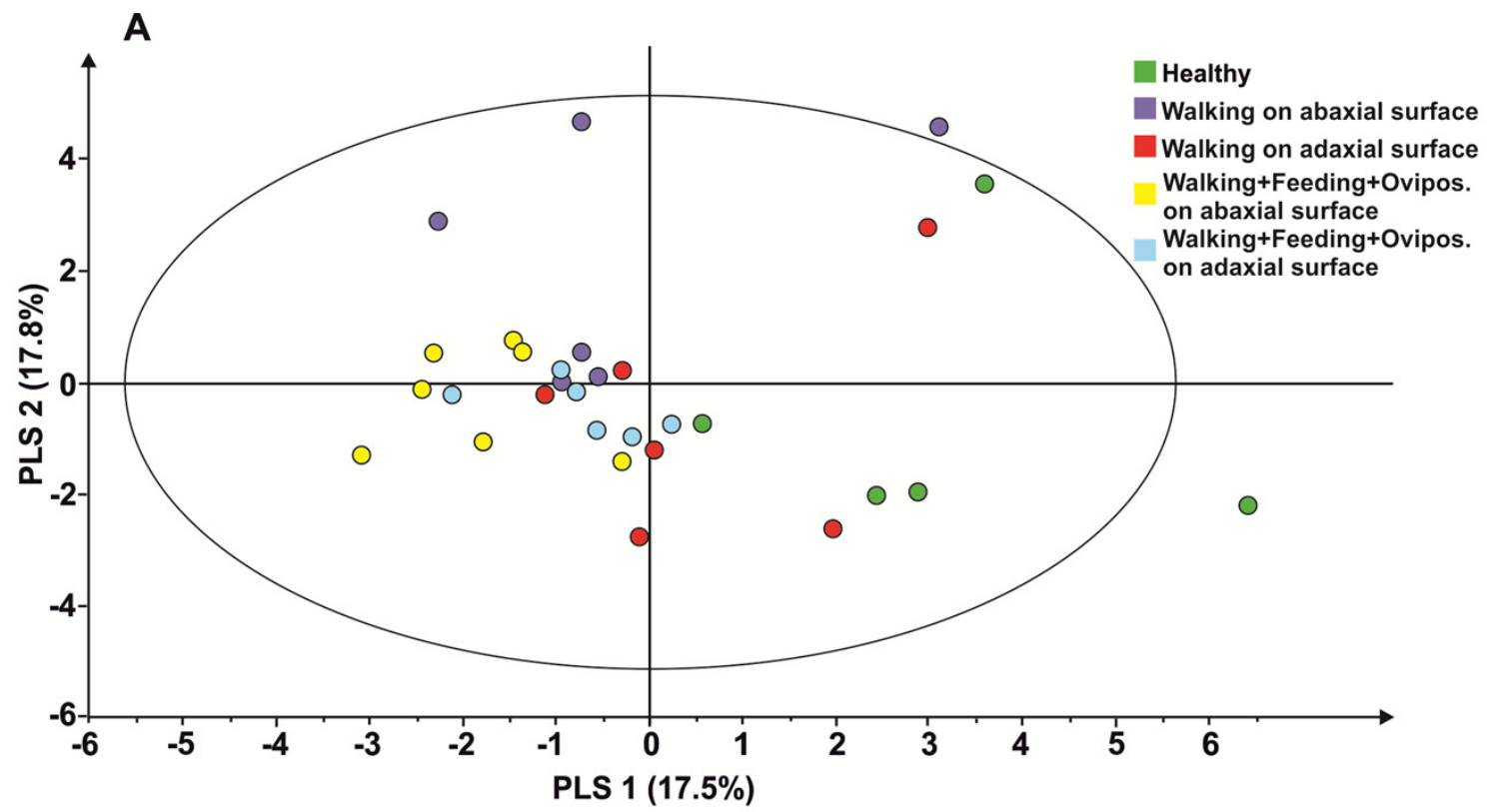

B

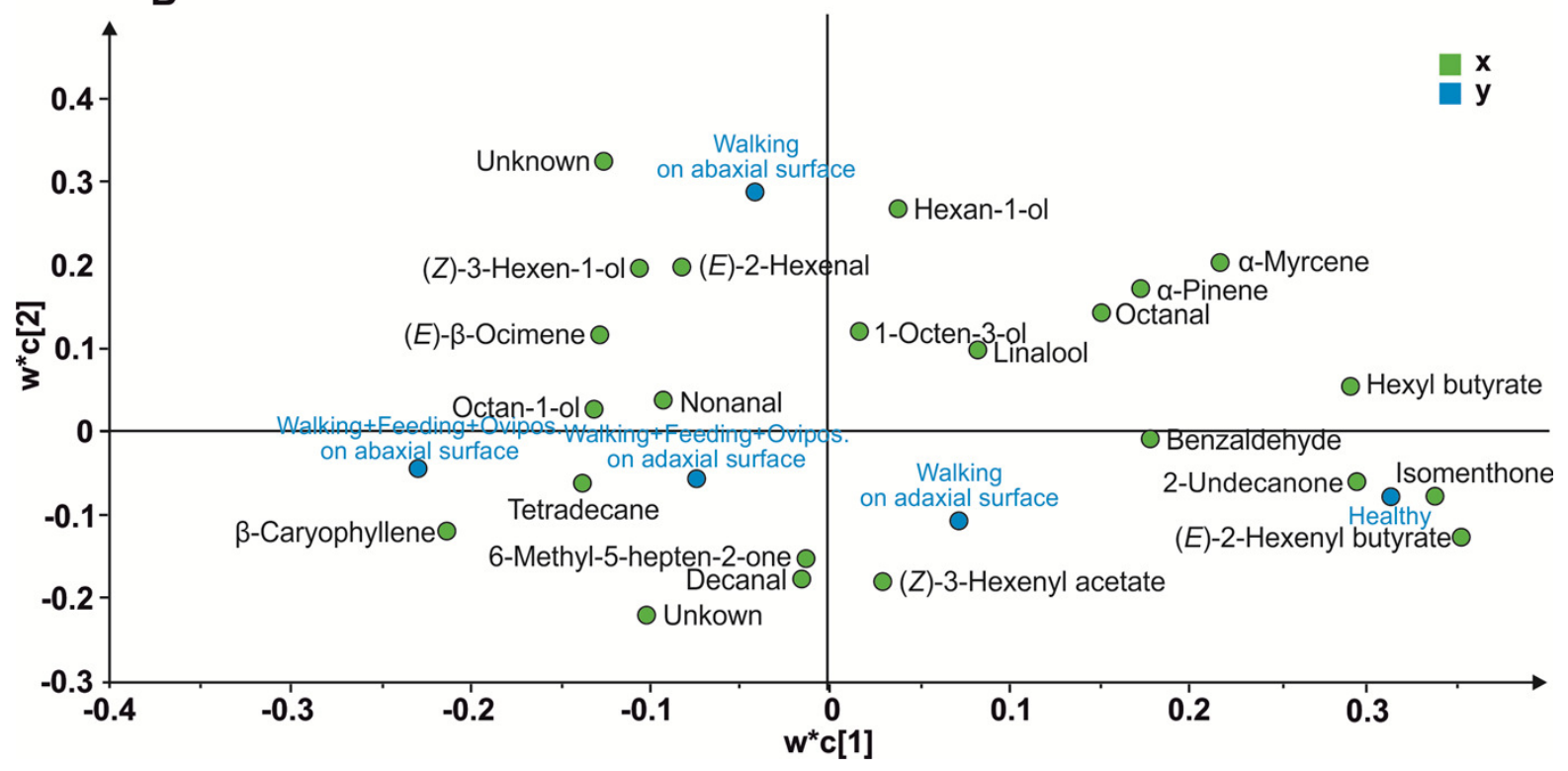

592 